\title{
BMJ Open Protocol for a systematic review on tertiary prevention interventions for patients with stroke in African countries
}

\author{
Eric Sven Kroeber (D) , ${ }^{1}$ Lucas Adam, ${ }^{1}$ Adamu Addissie, ${ }^{2}$ Alexander Bauer, ${ }^{1}$ \\ Thomas Frese, ${ }^{1}$ Eva Johanna Kantelhardt (D) ," Susanne Unverzagt (D) ${ }^{1,4}$
}

To cite: Kroeber ES, Adam L, Addissie A, et al. Protocol for a systematic review on tertiary prevention interventions for patients with stroke in African countries. BMJ Open 2020;10:e038459. doi:10.1136/ bmjopen-2020-038459

- Prepublication history for this paper is available online. To view these files, please visit the journal online (http://dx.doi. org/10.1136/bmjopen-2020038459).

Received 11 March 2020 Revised 03 August 2020 Accepted 04 August 2020

\section{Check for updates}

(c) Author(s) (or their employer(s)) 2020. Re-use permitted under CC BY-NC. No commercial re-use. See rights and permissions. Published by BMJ.

${ }^{1}$ Center of Health Sciences, Institute of General Practice and Family Medicine, Martin-LutherUniversitat Halle-Wittenberg, Halle, Sachsen-Anhalt, Germany

${ }^{2}$ Preventive Medicine, Addis Ababa University School of Public Health, Addis Ababa, Oromia, Ethiopia

${ }^{3}$ Center of Health Sciences, Institute for Medical

Epidemiology, Biostatistics and Informatics, Martin-LutherUniversitat Halle-Wittenberg, Halle, Sachsen-Anhalt, Germany

${ }^{4}$ Department of General Practice, Leipzig University, Leipzig,

Sachsen, Germany

Correspondence to

Dr Susanne Unverzagt;

susanne.unverzagt@uk-halle.de

\section{ABSTRACT}

Introduction Stroke is one of the common causes of mortality, morbidity and years of life lost worldwide. Baseline research on stroke epidemiology, prevention, acute and rehabilitative interventions in Africa is necessary to approach specific contexts and regional circumstances. Most studies on stroke have been conducted in highincome countries. This protocol describes the methodology to summarise the best available evidence on tertiary preventive strategies like rehabilitation interventions for patients with stroke in African contexts.

Methods and analysis We will include experimental studies and prospective cohort studies conducted in African countries. A protocol has been registered in PROSPERO. Systematic search will include eight electronic databases (MEDLINE, Embase, the Cochrane Library, CINAHL, Cab-Direct, Physiotherapy Evidence Database (PEDro), African Journals Online and African Index Medicus) and the International Clinical Trials Register Platform and base on predefined search terms. We will search from inception of each database and repeat this strategy 3 months prior to review submission. Details of all eligible studies will be extracted and risk of bias for outcomes on global disability or dependence in daily living will be assessed. Main aim of this systematic review is to provide a narrative description of evidence on tertiary prevention strategies (including rehabilitation) for stroke. This description will be visualised in structured tables to aid interpretation of study characteristics, intervention effects and certainty of the evidence.

Ethics and dissemination No ethical approval is necessary. Results will be presented in national and international conferences and published in a peerreviewed journal.

PROSPERO registration number CRD42020159125.

\section{INTRODUCTION}

\section{Background}

Worldwide, stroke was the second most common cause of death and the third most common cause of morbidity in 2016. ${ }^{1}$ The number of years lived with disability was estimated to be 18.7 million in $2017 .^{2}$ In the African region, the number of patients with

\section{Strengths and limitations of this study}

- The planned systematic review will summarise the best available evidence on tertiary prevention strategies (including rehabilitation) for patients with stroke in African countries.

- Inclusion criteria are restricted to experimental studies and high-quality prospective cohort studies conducted in African countries with no restrictions on language and time of publication.

- Systematic search will be conducted in eight electronic databases (MEDLINE, Embase, the Cochrane Library, CINAHL, Cab-Direct, Physiotherapy Evidence Database (PEDro), African Journals Online and African Index Medicus) and the International Clinical Trials Register Platform to get a comprehensive overview on all investigated interventions.

- Results will be narratively described and visually presented in structured tables to visualise the effect size of included interventions and to demonstrate effects on global disability or dependence in daily living outcomes.

- Despite the restriction to the African context, we expect a high grade of diversity among eligible studies regarding methodology, participants, settings, interventions and outcomes studied that may limit the overall generalisability.

stroke has risen from the 10th rank among the most common causes of morbidity in 2000 with a total of 297600 deaths to rank 7 with 373500 deaths in $2016 .{ }^{1}$ Stroke accounted for one of the leading causes of years of life lost in $2017 .^{3}$

Medical care of patients with stroke in African countries generally lacks diagnostic resources, acute management options, implemented rehabilitative and preventive strategies and awareness among the population. ${ }^{4-6}$ The implementation of cost-effective interventions might profoundly reduce mortality and limit economic losses. ${ }^{7}$ Tackling and reducing the burden of high premature mortality due to non-communicable diseases (NCDs) through prevention and treatment 
have been designated as a goal within the UN 2030 Agenda (Target 3.4) ${ }^{8}$ Evidence is needed that reflects regional differences in NCD incidences, their increase over time due to better diagnosis and changing risk factors of different populations in transition all located on the African continent. This requires research on interventions in appropriate settings, organised around the needs and expectations of people.

Baatiema et al summarised the existing evidence on acute stroke interventions generated in African countries in a 2017 systematic review. ${ }^{9}$ The main acute ischaemic stroke therapy options include systemic thrombolysis and mechanical thrombectomy. ${ }^{10}$ Acute haemorrhagic stroke treatment options include drug therapies to reduce blood pressure and to control haemostasis as well as surgical options. ${ }^{10-13}$ In addition to and following acute therapy, tertiary interventions are important measures to support patients in regaining functioning and returning into daily life at home away from hospital settings. ${ }^{14}$

\section{Description of the interventions and how they might work}

The upcoming review focuses on tertiary preventive stroke treatment strategies. The general aim of tertiary prevention interventions is the reduction of the severity and secondary complications of an already established disease in individuals. ${ }^{15}$ Besides rehabilitation treatments, it includes strategies that aim to prevent activity limitations and to promote independence, participation and inclusion. ${ }^{16}$ In case of stroke, the treatment strategies try to make use of the long-lasting postlesional cerebral plasticity starting at day 1 after stroke onset in the subacute phase in order to improve and regain the functioning of stroke survivors. ${ }^{17} 18$ Additionally, restitution of noninfarcted penumbral areas, resolution of diaschisis and behavioural compensation are discussed to have a major role in functional recovery. ${ }^{19}$

Tertiary prevention includes rehabilitation measures like occupational therapy, physiotherapy, speech and language therapy and target the individual needs of patients with their specific functioning deficits. ${ }^{14} 2021$ These therapeutic strategies are complemented by other measures including exercise, compensative, psychosocial and lifestyle interventions. Exercise therapy is especially used to support patients in regaining motor functioning. ${ }^{22}$ Compensative strategies comprise techniques to be learnt by patients who are used to overcome obstacles of daily life if functioning cannot be fully regained and thusly support partial recoveries. ${ }^{14}$ Psychosocial interventions are used to support patients and their relatives in order to adjust to the psychological challenges of living with stroke-related disabilities. ${ }^{23}$ Lifestyle interventions consist of strategies like the implementation of nutrition, physical activity and self-management strategies tailored to individual patient needs. ${ }^{2425}$

Though oftentimes long lasting, tertiary preventive interventions have shown great clinical impact and considerably reduce long-term disability and morbidity of patients with stroke. ${ }^{14}$

\section{Statement of purpose}

Many successful stroke management strategies and therapies were developed over the last decades. However, evidence from high-quality prospective trials that address the specific characteristics of African patients and consider limited resources are still lacking. ${ }^{926}$ There is a demand of well-informed solutions to formulate effective health agendas for prevention and therapy and improvement of availability and affordability of treatment options for cardiovascular diseases including stroke. ${ }^{27}$

The main aim of this systematic review is a summary of the best available evidence on benefits and harms of tertiary prevention interventions for patients with stroke in African countries to reduce disability and dependence in daily living. This protocol specifies inclusion criteria for study design, participants, interventions and outcomes, search methods, study selection, data extraction, data synthesis as well as the investigation of potential effect modifiers as a basis of a systematic review.

\section{METHODS AND ANALYSIS}

This systematic review will follow the recommendations of the Preferred Reporting Items for Systematic Reviews and Meta-Analyses and their and protocols. ${ }^{28} 29$ The methods are described in the Cochrane Handbook for Systematic Reviews of Interventions. ${ }^{30}$ The protocol is registered in the International Prospective Register of Systematic Reviews.

\section{Inclusion criteria}

Types of studies

We will include experimental studies (individual randomised controlled trials (RCTs), cluster-RCTs, randomised cross-over trials, non-randomised trials) and observational studies initiated and conducted in African countries. We will restrict observational studies to prospective cohort studies and use the algorithm ${ }^{31}$ with a control of most important potential confounders (eg, age, stroke severity, ${ }^{32}$ time after onset ${ }^{18}{ }^{32}$ ) to minimise biassed treatment effects.

Eligible trials should be published as full-text publications according to the Consolidated Standards of Reporting Trials, ${ }^{29}$ Standard Protocol Items: Recommendations for Interventional Trials ${ }^{33}$ or the Strengthening the Reporting of Observational Studies in Epidemiology statement. ${ }^{34}$ We will exclude international multicentr studies with less than $50 \%$ of sites in African countries as well as retrospective and non-controlled studies.

\section{Types of participants}

Studies on adult patients with a clinical diagnosis of stroke will be included (including ischaemic stroke and intracerebral haemorrhage). We will exclude studies that included solely children and adolescents as well as studies on subarachnoid haemorrhage patients (table 1). We will differentiate between patients being treated in the acute stroke ( $\leq 48$ hour after stroke onset), subacute stroke ( $>48$ 
Table 1 Inclusion criteria

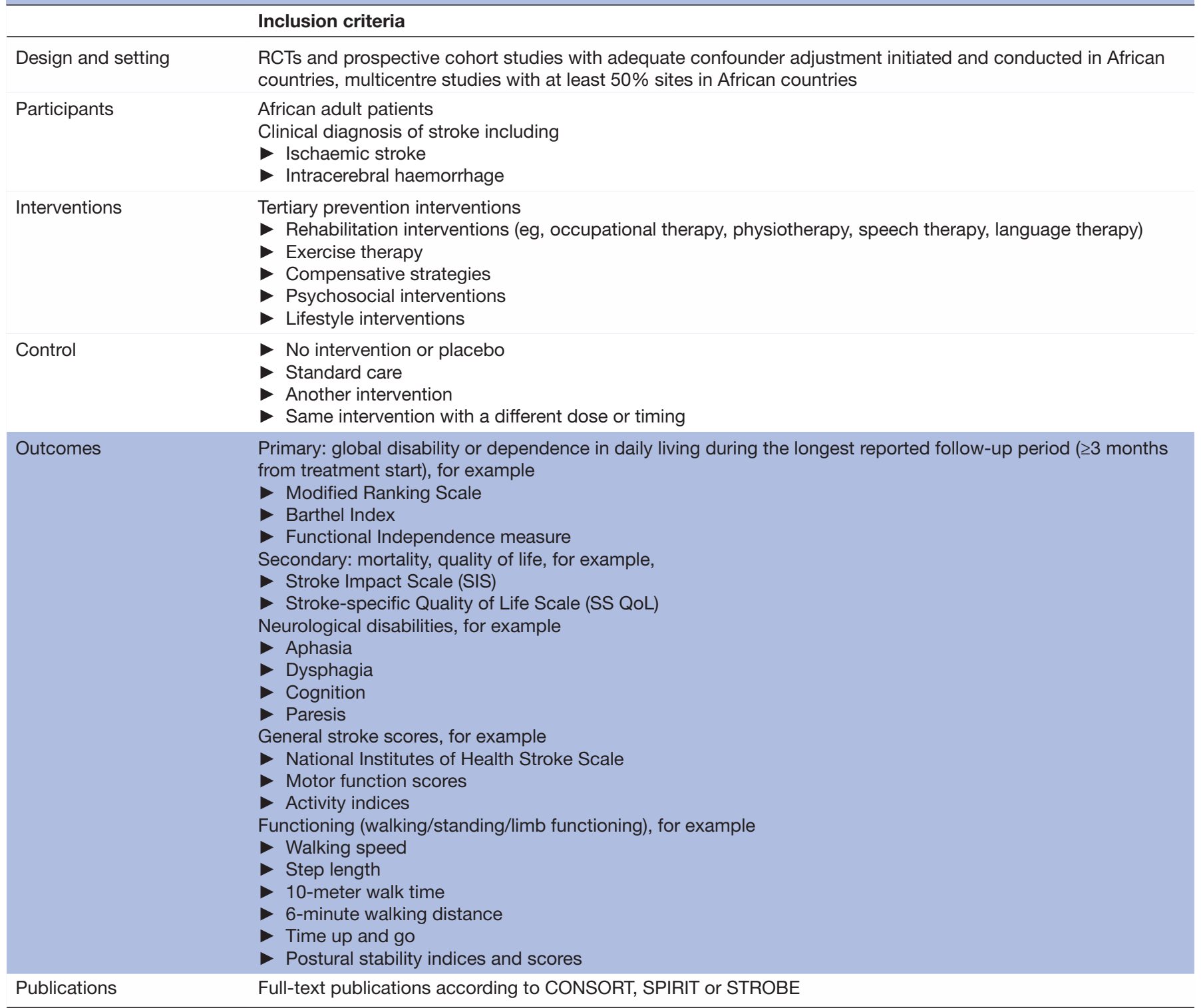

CONSORT, Consolidated Standards of Reporting Trials; RCT, randomised controlled trial; SPIRIT, Standard Protocol Items: Recommendations for Interventional Trials; STROBE, Strengthening the Reporting of Observational Studies in Epidemiology.

to $\leq 6$ months after stroke onset) and chronic stroke ( $>6$ months after stroke onset). ${ }^{18}$

\section{Types of interventions}

Interventions will cover all tertiary prevention interventions that aim to reduce global disabilities and dependence in daily living. Interventions may include different rehabilitation interventions (eg, occupational therapy, physiotherapy, speech and language therapy) as well as exercise therapy, compensative and psychosocial strategies and lifestyle modifications.

Interventions like exercise therapy and lifestyle interventions may transcend the border between tertiary and secondary prevention. Contrary to tertiary prevention, secondary prevention aims to mediate common risk factors after a first-ever stroke in order to prevent a second stroke, ${ }^{12}$ secondary prevention interventions exemplarily try to slow down the development atherosclerosis or to control hypertension that are precursors of stroke. ${ }^{12}{ }^{13} \mathrm{We}$ will only include studies with the mainly tertiary preventive aims and exclude studies that are solely targeting secondary preventive aims.

Control groups may cover no intervention, placebo, standard care, another (less intensive) intervention or the same intervention with a different dose or timing (table 1).

\section{Types of outcomes}

Primary outcomes are patient-relevant outcomes describing global disability or dependence in daily living (eg, Modified Rankin Scale, Barthel Index or Functional Independence Measure) after a minimum of 3-month follow-up over the longest reported follow-up period. Secondary outcomes include mortality, quality of life, 
neurological disabilities, general stroke scores and functioning (table 1).

\section{Systematic search for the identification of relevant studies}

Searches will be conducted in eight electronic databases (MEDLINE, Embase, the Cochrane Library, CINAHL, Cab-Direct, Physiotherapy Evidence Database (PEDro), African Journals Online and African Index Medicus) and registers of ongoing and completed trials in the International Clinical Trials Register Platform (http://apps.who. int/trialsearch/AdvSearch.aspx). The search starts at inception of each database and will be repeated 3 months prior to the date of review submission. Search strings will include Medical Subject Headings (MESH) and terms that describe stroke (eg, cerebrovascular, cerebral or intracranial accidents, infarction, disease, haemorrhage or ischaemia), Africa and a list of all 55 African countries and terms related to eligible study designs. To identify additional ongoing, published or unpublished studies, we will screen the reference lists of all included full-text papers and contact specialists from the Stroke Investigative Research \& Education Network. ${ }^{35}$ No language limits will be applied.

Online supplemental file 1 presents the search strategy in Medline (Ovid). We will adapt the strategy to the search in other databases and document the search process to the extent that all database searches are reproducible. All references will be exported into a reference management software (Endnote). Duplicate references will be deleted. Duplicate references will be identified in case of congruence of authors, title, year and journal (volume and pages) and deleted.

\section{Study selection}

Two independent authors (one physician and one methodologist) will screen all titles and abstracts from the systematic search and choose potentially eligible references in Rayyan. ${ }^{36}$ They will retrieve the full-text publications, screen all full texts, identify studies for inclusion and record reasons for exclusion. Two authors will discuss all differences in screening of title and abstract and full texts. Any disagreement will be resolved through discussion or, if required, consultation with a third author. Titles and references published in other languages and full-text publications of potentially references will be translated by a translation service.

We will critically check all studies for duplicate publication and name the studies on the basis of the first full-text publication of the primary outcome of the study. Authors from Addis Ababa University, Ethiopia with expertise in the field will be closely involved in all discussions.

\section{Data extraction}

One author will extract details to describe study design, population, interventions, outcomes and main results by using an assessment form, especially designed for this systematic review and piloted for the first five eligible studies. A second author will check all extractions. If study results are reported in more than one publication with conflicting results, the first full-text publication will be the main data source.

The data extraction form will include information on

- Publication: study name and reference.

- Study design and setting: country, urban or rural area, period for inclusion and longest follow-up period.

- Population: inclusion and exclusion criteria (including diagnostic criteria), sample size and baseline characteristics: age, sex, type of stroke (infarction vs haemorrhagic), preplanned acute treatment, stroke severity, side of haemiplegia, time from onset of stroke to randomisation.

- Intervention and control group: description of the active component to describe the difference between groups (detailed description of intervention, duration of intervention, profession of care givers), number of randomised patients per group, for non-randomised and observational studies with a description of confounder adjustment.

- Outcomes (primary, secondary) with period of follow-up.

- Main results on preplanned outcomes of the systematic reviews (number of analysed cases with mean and SD or absolute and relative frequencies per group) and reported intervention effects.

- Possible sources of heterogeneity (eg, diagnostic criteria, urban or rural setting, study design).

Results on metric data will base on the precision of reported values and resulting intervention effects. Results on dichotomous data will be described as relative frequencies and resulting relative risks (RRs). Results on metric data will be reported with mean and SD or median and ranges and resulting mean differences (MDs). All treatment effects will be reported with their corresponding 95\% CIs (95\% CI).

We will calculate MDs and standardised MDs (SMDs) and their 95\% CI from reported treatment effects or estimate these on the basis of reported means, SD and samples sizes ${ }^{37}$ or medians, ranges and sample sizes of intervention groups. ${ }^{38}$ We will calculate RRs and ORs from reported treatment effects or recalculate these treatment effects from reported numbers of events and nonevents in the intervention groups. Generally, we prefer results from adjusted analyses.

For cluster-RCTs, we will calculate an inflated SE that accounts for clustering, for cross-over RCTs, we will use results from the first period. ${ }^{39}$

\section{Assessment of risk of bias in included studies}

Risk of bias will be described and judged independently by two authors. We will use the revised Cochrane risk of bias tool for RCTs ${ }^{40}$ or the risk of bias in non-randomised studies of interventions tool ${ }^{41}$ to assess risk of bias for results on the preplanned primary outcome. For RCTs, we will judge bias in five different domains arising from the randomisation process, due to deviations from intended interventions, missing outcome data, measurement of 
the outcomes and selection of the reported results on the basis of predefined signalling questions ${ }^{30}$ and summarise overall bias as 'low', 'some concerns' or 'high' ${ }^{30}$ For nonrandomised studies, we will judge risk of bias in seven domains and differentiate bias due to confounding, selection of study participants, classification of interventions, deviations from intended interventions, missing data, measurement of outcomes and selection of reported results. We will summarise overall bias as 'low', 'moderate', 'serious' or 'critical'. ${ }^{41}$ We will summarise risk of bias in two tables to illustrate these findings. Discrepancies will be resolved by discussion between the authors or with a third author until consensus is obtained. If this is not possible, authors of primary trials will be contacted.

\section{Data synthesis}

Main aim of our systematic review is to provide a narrative overview of the best available evidence on interventions for stroke. We will present a structured tabulation of studies and order the studies in subgroups of comparable interventions of different tertiary preventive strategies, study design and study size.

Treatment effects and their $95 \%$ CI for the primary outcome will be visually displayed in the form of forest plots. Even for comparable studies, we do not expect one single study effect and will apply a random effects model. ${ }^{42}$ RRs are the preferred measure of the relative effect for binary-reported outcomes and MDs for metric outcomes. However, if different studies use different instruments to assess the same outcome, SMDs or ORs will be used to combine results of different studies. ${ }^{37}$ Data will be analysed using Review Manager V.5.3. ${ }^{43}$

Management recommendations in clinical guidelines primarily focus on absolute effects on patient-important outcomes. Therefore, we will additionally present absolute changes if adequate information on baseline risk in the control group is available. ${ }^{44}$

Publication bias will be investigated by funnel plots, funnel plot tests, suitable tests for small-study effects ${ }^{45}$ and treatment effect estimates adjusted for small study effects. ${ }^{46}$ We will use funnel plots, funnel plot test and adjustments in comparisons with at least 10 included studies with the same outcome.

\section{Investigation of heterogeneity}

This systematic review will merge material with an element of diversity differing in methodology, participants, setting, interventions or outcome studied. The $\mathrm{I}^{2}$ statistics will be used to assess heterogeneity as considerable, substantial, moderate or not important. Differences will be described in subgroup analyses and the following sources of heterogeneity will be considered:

- Differences in methodological quality of studies (RCTs vs non-RCTs).

- Different setting (urban vs rural, Northern Africa vs sub-Saharan Africa).

- Differences in timepoint of treatment (acute vs subacute vs chronic care).

\section{Patient and public involvement}

Scientists from one African country (School of Public Health, Addis Ababa University, Ethiopia) and stroke specialists from the Human Heredity \& Health in Africa consortium will be involved in the development of the review. ${ }^{35}$ No patient or members of the public will be involved. However, results might be a basis to plan local intervention studies and to generate specific guideline recommendations.

\section{DISCUSSION}

Worldwide, NCDs accounted for $73.4 \%$ (41.1 million) of all deaths and $79.5 \%$ of years lived with a disability in 2017. ${ }^{2}{ }^{3}$ Low-income and middle-income countries (LMICs) suffer from a double disease burden due to the epidemiological transition from infectious to NCDs with a relevant shift in causes of illnesses, disability and deaths. ${ }^{3}$ Due to the large burden of infectious diseases and other competing issues, NCDs were not a prioritised public health agenda in many African countries over the last decades. ${ }^{47-49}$ Rapidly growing prevalence rates, early ages of onset and devastating outcomes in LMICs resulted in changing public health agenda priorities. ${ }^{4} 7275051$

Several studies on stroke have been conducted in African countries and were published in recent years. ${ }^{9} 26$ Baatiema et al summarised evidence on acute stroke interventions generated in African countries in a 2017 systematic review. ${ }^{9}$ A systematic review by Dee et al analysed stroke rehabilitation outcomes in LMICs. They included eight clinically controlled studies and six cohort studies conducted in African countries, but only two RCTs. ${ }^{26}$ In a piloting search in three databases (Medline, CENTRAL and CINAHL) on 1 December 2019, we were able to identify a total of 17 eligible RCTs. Given the discrepancy of the number of the RCTs found in our recherche, and on the existing systematic review, we decided to initiate a comprehensive review on tertiary preventive stroke interventions in Africa, relying on RCTs and confounder controlled prospective cohort studies to provide the best available evidence.

Our synthesis of the existing evidence on tertiary preventive interventions will aid health professionals and researchers to find and work with up-to-date knowledge on stroke care in African settings. The review will help to address the current knowledge gaps and unexplored research fields and to identify resources of application of evidence-based interventions. This may support the formulation of strategies to strengthen clinical and preventive capacities of current healthcare systems in African countries.

Contributors ESK has written the draft of this protocol and submitted this protocol, responded to reviewer comments. LA contributed to the development of the inclusion criteria, data extraction and search strategy for stroke and neurological disorders and has written the draft of the protocol, responded to reviewer comments. AA provided expertise on the needs of evidence in the African context. AB critically read and commented the drafted protocol. TF critically read and commented the drafted protocol and provided expertise on primary care. EJK 
provided expertise on the needs of evidence in the African context and commented the protocol. SU has expertise in systematic reviews and is the guarantor of the methodological quality of this systematic review, has written the draft, responded to reviewer comments. All authors discussed the comments of the reviewers and approved of the final revised manuscript version.

Funding The authors have not declared a specific grant for this research from any funding agency in the public, commercial or not-for-profit sectors.

Competing interests None declared.

Patient and public involvement Patients and/or the public were not involved in the design, or conduct, or reporting, or dissemination plans of this research.

Patient consent for publication Not required.

Provenance and peer review Not commissioned; externally peer reviewed.

Open access This is an open access article distributed in accordance with the Creative Commons Attribution Non Commercial (CC BY-NC 4.0) license, which permits others to distribute, remix, adapt, build upon this work non-commercially, and license their derivative works on different terms, provided the original work is properly cited, appropriate credit is given, any changes made indicated, and the use is non-commercial. See: http://creativecommons.org/licenses/by-nc/4.0/.

\section{ORCID iDs}

Eric Sven Kroeber http://orcid.org/0000-0002-7201-6023

Eva Johanna Kantelhardt http://orcid.org/0000-0001-7935-719X

Susanne Unverzagt http://orcid.org/0000-0002-0108-0415

\section{REFERENCES}

1 Organization WH. Global health estimates 2016: deaths by cause, age, sex, by country and by region, 2000-2016. Geneva: WHO, 2019.

2 GBD 2017 Disease and Injury Incidence and Prevalence Collaborators. Global, regional, and national incidence, prevalence, and years lived with disability for 354 diseases and injuries for 195 countries and territories, 1990-2017: a systematic analysis for the global burden of disease study 2017. Lancet 2018;392:1789-858.

3 GBD 2017 Causes of Death Collaborators. Global, regional, and national age-sex-specific mortality for 282 causes of death in 195 countries and territories, 1980-2017: a systematic analysis for the global burden of disease study 2017. Lancet 2018;392:1736-88.

4 Johnson W, Onuma O, Owolabi M, et al. Stroke: a global response is needed. Bull World Health Organ 2016;94:634-634A.

5 Owolabi MO, Akarolo-Anthony S, Akinyemi R, et al. The burden of stroke in Africa: a glance at the present and a glimpse into the future. Cardiovasc J Afr 2015;26:S27-38.

6 Owolabi M, Johnson W, Khan T, et al. Effectively combating stroke in low- and middle-income countries: placing proof in pragmatismThe Lancet neurology Commission. J Stroke Med 2018;1:65-7.

7 Juma K, Juma PA, Mohamed SF, et al. First Africa noncommunicable disease research conference 2017: sharing evidence and identifying research priorities. J Glob Health 2019;8:020301.

8 Nations U. Sustainable development goals. goal 3: ensure healthy living and promote well-being for all at all ages, 2019. Available: https://www.un.org/sustainabledevelopment/health/;

9 Baatiema L, Chan CKY, Sav A, et al. Interventions for acute stroke management in Africa: a systematic review of the evidence. Systematic Reviews 2017;6:213.

10 Powers WJ, Rabinstein AA, Ackerson T, et al. Guidelines for the early management of patients with acute ischemic stroke: 2019 update to the 2018 guidelines for the early management of acute ischemic stroke: a guideline for healthcare professionals from the American Heart Association/American Stroke Association. Stroke 2019;50:e344-418.

11 Hemphill III JC, Greenberg SM, Anderson CS, et al. Guidelines for the management of spontaneous intracerebral hemorrhage: a guideline for healthcare professionals from the American Heart Association/ American Stroke Association. Stroke 2015;46:2032-60.

12 Kernan WN, Ovbiagele B, Black HR, et al. Guidelines for the prevention of stroke in patients with stroke and transient ischemic attack: a guideline for healthcare professionals from the American Heart Association/American Stroke Association. Stroke 2014;45:2160-236.

13 Dengler R, Diener $\mathrm{H}-\mathrm{C}$, Schwartz A, et al. Early treatment with aspirin plus extended-release dipyridamole for transient ischaemic attack or ischaemic stroke within $24 \mathrm{H}$ of symptom onset (early trial): a randomised, open-label, blinded-endpoint trial. Lancet Neurology 2010;9:159-66.
14 Winstein CJ, Stein J, Arena R, et al. Guidelines for adult stroke rehabilitation and recovery: a guideline for healthcare professionals from the American Heart Association/American Stroke Association. Stroke 2016;47:e98-169.

15 Kisling LA, Das JM. Prevention strategies. Treasure Island (FL): StatPearls Publishing, 2019.

16 Khasnabis C, Motsch K, Achu K, et al. Community-based rehabilitation: CBR guidelines. Geneva: World Health Organization, 2019.

17 Krakauer JW, Carmichael ST, Corbett D, et al. Getting neurorehabilitation right: what can be learned from animal models? Neurorehabil Neural Repair 2012;26:923-31.

18 Zhao L-R, Willing A. Enhancing endogenous capacity to repair a stroke-damaged brain: an evolving field for stroke research. Prog Neurobiol 2018;163-164:5-26.

19 Kwakkel G, Kollen B, Lindeman E. Understanding the pattern of functional recovery after stroke: facts and theories. Restor Neurol Neurosci 2004;22:281-99.

20 Platz T. Evidence-based guidelines and clinical pathways in stroke rehabilitation-an international perspective. Front Neurol 2019;10:200.

21 Nilsen D, Gillen G, Arbesman M, et al. Occupational therapy interventions for adults with stroke. Am J Occup Ther 2015;69:6905395010p1-3.

22 Caspersen CJ, Powell KE, Christenson GM. Physical activity, exercise, and physical fitness: definitions and distinctions for healthrelated research. Public health rep 1985;100:126-31.

23 Kirkevold M, Kildal Bragstad L, Bronken BA, et al. Promoting psychosocial well-being following stroke: study protocol for a randomized, controlled trial. BMC psychology 2018;6:12.

24 Hill VA, Vickrey BG, Cheng EM, et al. A pilot trial of a lifestyle intervention for stroke survivors: design of healthy eating and lifestyle after stroke (HEALS). J Stroke Cerebrovasc Dis 2017;26:2806-13.

25 Sarrafzadegan Net al. Do lifestyle interventions work in developing countries? findings from the Isfahan healthy heart program in the Islamic Republic of Iran. Bull World Health Organ 2009;87:39-50.

26 Dee M, Lennon O, O'Sullivan C. A systematic review of physical rehabilitation interventions for stroke in low and lower-middle income countries. Disabil Rehabil 2020;42:473-501.

27 Mensah GA, Roth GA, Sampson UK, et al. Mortality from cardiovascular diseases in sub-Saharan Africa, 1990-2013: a systematic analysis of data from the global burden of disease study 2013. Cardiovasc J Afr 2015;26:S6-10.

28 Moher D, Liberati A, Tetzlaff J, et al. Preferred reporting items for systematic reviews and meta-analyses: the PRISMA statement. PLoS Med 2009;6:e1000097.

29 Moher D, Shamseer L, Clarke M, et al. Preferred reporting items for systematic review and meta-analysis protocols (PRISMA-P) 2015 statement. Systematic Reviews 2015;4:1.

30 Higgins J, Thomas J, Chandler J. Cochrane handbook for systematic reviews of interventions version 6.0, 2019.

31 Seo H-J, Kim SY, Lee YJ, et al. A newly developed tool for classifying study designs in systematic reviews of interventions and exposures showed substantial reliability and validity. J Clin Epidemiol 2016;70:200-5.

32 Kwakkel G, Lannin NA, Borschmann K, et al. Standardized measurement of sensorimotor recovery in stroke trials: consensus-based core recommendations from the stroke recovery and rehabilitation roundtable. Neurorehabil Neural Repair 2017;31:784-92.

33 Chan AW, Tetzlaff JM, Altman DG, et al. SPIRIT 2013 statement: defining standard protocol items for clinical trials. Ann Intern Med 2013;158:200-7.

34 von Elm E, Altman DG, Egger M, et al. The strengthening the reporting of observational studies in epidemiology (STROBE) statement: guidelines for reporting observational studies. Ann Intern Med 2007;147:573-7.

35 Owolabi M, Ovbiale B. Stroke investigative research \& educational network (SIREN), 2020. Available: https://h3africa.org/index.php/ consortium/stroke-investigative-research-educational-network-siren/ [Accessed 17 Jun 2020].

36 Ouzzani M, Hammady H, Fedorowicz Z, et al. Rayyan-a web and mobile app for systematic reviews. Systematic Reviews 2016;5:210.

37 Borenstein M, Hedges LV, Higgins JPT, et al. Introduction to metaanalysis, 2009.

38 Hozo SP, Djulbegovic B, Hozo I. Estimating the mean and variance from the median, range, and the size of a sample. BMC Med Res Methodol 2005;5:13.

39 Higgins JP, Eldridge S, Li T. Including variants on randomized trials. In: Cochrane handbook for systematic reviews of interventions, 2019: 569-93. 
40 Sterne JA, Savović J, Page MJ, et al. RoB 2: a revised tool for assessing risk of bias in randomised trials. Bmj 2019;366:14898.

41 Sterne JA, Hernán MA, Reeves BC, et al. ROBINS-I: a tool for assessing risk of bias in non-randomised studies of interventions. Bmi 2016;355:i4919.

42 Higgins JPT, Green S. Cochrane handbook for systematic reviews of interventions version 5.1.0. Available: http://handbook-5-1.cochrane. org/; 2011

43 Centre TNC. Review Manager (RevMan) [Computer program]. Version 5.3, 2014.

44 Guyatt GH, Oxman AD, Santesso N, et al. GRADE guidelines: 12. Preparing summary of findings tables-binary outcomes. J Clin Epidemiol 2013;66:158-72.

45 Egger M, Davey Smith G, Schneider M, et al. Bias in meta-analysis detected by a simple, graphical test. Bmj 1997;315:629-34.

46 Shi L, Lin L. The trim-and-fill method for publication bias: practical guidelines and recommendations based on a large database of meta-analyses. Medicine 2019;98:e15987.
47 Misganaw A, Mariam DH, Araya T. The double mortality burden among adults in Addis Ababa, Ethiopia, 2006-2009. Prev Chronic Dis 2012;9:E84

48 Dzudie A, Rayner B, Ojji D, et al. Roadmap to achieve 25\% hypertension control in Africa by 2025. Cardiovasc J Afr 2017;28:262-72.

49 EPHI. Steps survey on risk factors for non-cimmunicable diseases and prevalence of selected NCDs, Ethiopia - summary report. Addis Ababa: Ethiopian Public Health Institute, 2016.

50 Ahmed R, Robinson R, Mortimer K. The epidemiology of noncommunicable respiratory disease in sub-Saharan Africa, the Middle East, and North Africa. Malawi Med. J 2017;29:203-11.

51 Arokiasamy P,Kowal P, capistrant BD, et al . Chronic noncommunicable diseases in 6 low- and middle-income countries: findings from wave 1 of the world health organization's study on global ageing and adult health (SAGE). Am J Epidemiol 2017;185:414-28. 